\title{
Influence of patella height after patella fracture on clinical outcome: a 13-year period
}

\author{
Pesch Sebastian $^{1} \cdot$ Zyskowski Michael $^{1} \cdot$ Greve Frederik $^{1} \cdot$ Müller Michael $^{1} \cdot$ Wurm Marcus $^{1} \cdot$ Crönlein Moritz $^{1}$. \\ Biberthaler Peter ${ }^{1} \cdot$ Kirchhoff Chlodwig $^{1}$
}

Received: 17 July 2020 / Accepted: 23 March 2021 / Published online: 6 April 2021

(c) The Author(s) 2021

\begin{abstract}
Introduction The incidence of patella fracture is statistically low (0.5-1.5\%) compared to other fractures of the extremities [Patella fractures 76(10):987-997, 2005]. In the latter research, patella fractures if treated surgically present an overall inferior functional outcome. Little is known about the influence of the postoperative patella height on the clinical outcome. Therefore, the aim of our study was to analyse the influence of the patella height on the patients' functional outcome after surgery. Methods In this retrospective study the in-house trauma register of our level I University trauma center was screened for patients suffering patella fractures treated surgically. Patella height of the same patients was evaluated on lateral X-rays using the Insall-Salvati Ratio (ISR). The patients' X-rays were analyzed at two time points for the ISR, whereas group A presents ISR data right after surgery and group B data at the latest follow up (minimum 6 weeks). The change of mean ISR at both time points was tested for significance. The functional outcome was measured by the "Munich Knee Questionaire" (MKQ). These MKQ results of different patella heights and fracture types were compared.

Results The screening of our in-house trauma register revealed 375 patients between the years 2003 and 2016. Out of these 54 patients (34f, $20 \mathrm{~m}$ ) were enrolled. In detail the follow-up time for ISR between group A and B accounted for a mean of $503.8 \pm 655.7$ days. The MKQ was assessed at a mean of $1367.0 \pm 1042.8$ days after surgery. According to the AOclassification 10\% AO.34 type B and 90\% AO.34 type C fractures were found. Group A showed in 9.1\% a patella baja and in $27.3 \%$ a patella alta compared to group B presenting $20.0 \%$ patella baja and $14.5 \%$ patella alta. There was no significant difference in functional outcome referring to the MKQ in patella alta (MKQ 69.0\% \pm 18.2 ) or baja (MKQ 67.1\% \pm 17.9 ) $(p=0.9)$. No significant functional difference between AO34.type B (MKQ 74.5\% \pm 11.0 ) and AO34.type C fractures (MKQ $64.0 \% \pm 15.0)$ resulted $(p=0.1)$.
\end{abstract}

Conclusion Our results demonstrate that different postoperative patella heights apparently do not influence the functional outcome in the short follow-up.

Keywords Patella fracture $\cdot$ Comminuted patella fracture $\cdot$ Patella alta $\cdot$ Patella baja $\cdot$ Locking plate fixation $\cdot$ Patientoriented outcome measurement

\section{Abbreviations}

Patella baja

Patella alta

Patella norma

MKQ

IS

Inferior patella height

Elevated patella height

Normal patella height

Munich Knee Questionnaire

Insall-Salvati

Pesch Sebastian

sebastian.pesch@mri.tum.de

1 Department of Trauma Surgery, Klinikum Rechts Der Isar, Technical University of Munich, Ismaninger Strasse 22, 81675 Munich, Germany
ISR Insall-Salvati Ratio

n.s. Not significant

\section{Introduction}

Incidence of patella fractures is statistically low $(\leq 1 \%)$ when compared to [1] other extremity fractures, with a peak incidence in the 3rd and 6th decade of life [2]. Statistically male individuals suffer from patella fractures twice as much as women. Mostly a direct blunt trauma to the anterior surface of the patella causes transverse, longitudinal or comminuted fractures. Injuries to the patella resulting 
from acute extending movements in a flexed knee position are rare. Besides restoring the compromised functional knee-extending complex, indications for surgical treatment include a fracture gap of $2-3 \mathrm{~mm}$ and joint surface incongruence possibly resulting in posttraumatic osteoarthritis [3]. Transverse fractures of the patella encountering younger patients are mostly treated by surgery performing open reduction and internal fixation $[4,5]$. Tension band wiring with its different modifications (K-wires, figure-ofeight wiring, equatorial-cerclage) are well established in the surgical community [6]. Locking plate fixation apparently shows better biomechanical results in stress-loading tests in models and cadaver studies [6, 7]. Predictors for a good clinical outcome are a precise reconstruction of the joint surface, superior retention and finally healing of the fracture [8]. In the current literature, less than $50 \%$ of the patients present with good functional outcome following operative treatment $[9,10]$. Knowing the importance of the patella height for joint motion, there is a surprising lack of data of patella height on the postoperative clinical outcome following patella fracture. The low clinical and functional outcome after patella fractures is discussed in either biological aspects (pathologies of the synovia and/or Hoffa's fat pat) or referring in to biomechanical malformations due to, e.g., increased patella height with raised patella-femoral contact pressure during extensive movements [11-13]. Therefore, the aim of our study was to retrospectively analyze the influence of the postoperative patella height on the functional outcome following operatively treated patella fractures over a 13-year period.

\section{Materials and methods}

Between 2003 and 2016 a retrospective data analysis was conducted regarding patella fractures at our University levelI trauma center. Inclusion criteria were age between 18 and 85 years, patella fractures according to the $\mathrm{AO}$ classification, no concomitant knee injury and surgical treatment.

In general, the surgical procedure was adapted to the fracture type and actual soft tissue conditions. Several types of osteosynthesis such as tension band wiring and its different modifications (K-wires, figure-of-eight wiring, equatorialcerclage) were performed. Also locking plate fixation was done after its introduction to our trauma department in the year 2015.

Patella height was measured using the Insall-Salvati (IS) score on X-rays in lateral view. The Insall-Salvati Ratio (ISR) was assessed by measuring the distance from the tibial tuberosity to the inferior pole of the patella as well as by measuring the longest diameter of the patella (see Fig. 1). The ISR was assessed on X-rays of all enrolled patients primarily 2 days after surgery (group A) and secondarily

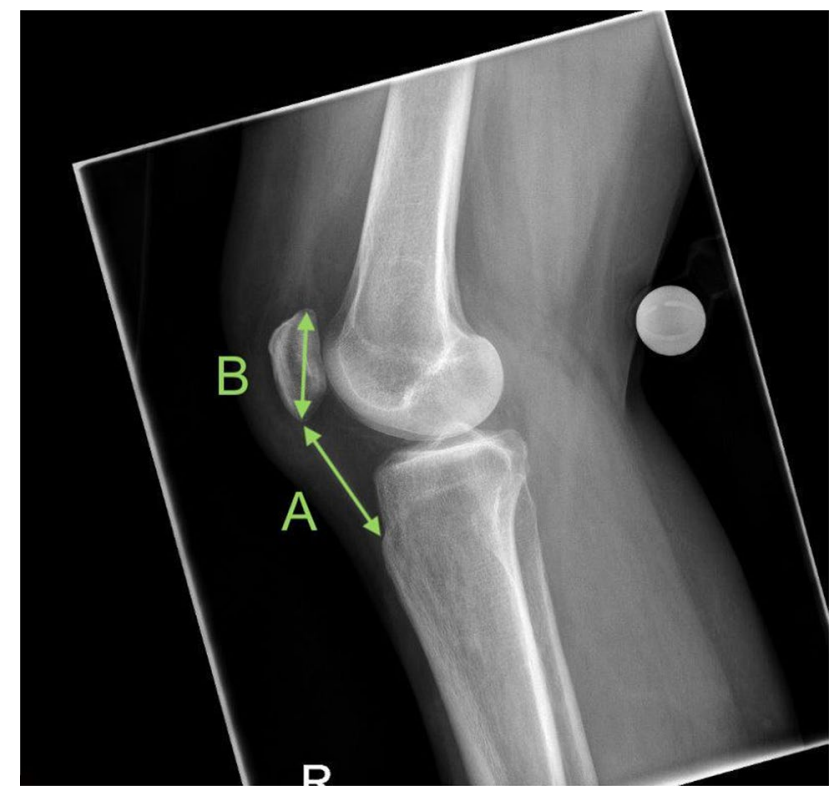

Fig. 1 Lateral view (X-ray) of the knee showing the assessment of the Insall-Salvati Ratio (ISR, A/B)

at the latest follow-up appointment (group B). The indication for X-rays at the latest follow-up was made upon the department's clinical standardized aftercare protocol (e.g., check for bone consolidation after 6 weeks, new complaints after consolidation or before implant removal). ISR-values between 0.8 and 1.2 were considered as normal, whereas in case of an ISR $<0.8$ a patella baja was described and correspondingly a patella alta was diagnosed if the ISR was $>1.2$ $[14,15]$. The Chi-square test was used to compare the ISR of group A and B for statistical significance.

The second part of the presented work was the analysis of the functional outcome using a standardized selfassessment questionnaire (Munich Knee Questionnaire (MKQ)) [16]. The MKQ as a self-assessment test combines well elaborated clinical tests for knee injuries (Knee Injury and Osteoarthritis Outcome Score (KOOS), International Knee Documentation Committee (IKDC), Western Ontario Meniscal Evaluation Tool (WOMET), Lysholm Score and Tegner activity score) [16]. In this context the functional outcome was analysed in refer to the patella height and the fracture type. The Kruskal-Wallis test was used to compare the MKQ of different patella heights in terms of patella baja, norma or alta. The Mann-Whitney test was used for non-parametric testing of significant difference in functional outcome in AO classification of AO.34 type B and C fractures. Data are provided as arithmetic mean and standard deviation. D'Agostino \& Pearson test showed a normal distribution of values. Statistical analysis was performed using the Sigma Stat 3.1 software (Systat Inc, Chicago, IL, USA) with a level of significance below $0.05(p<0.05)$. 


\section{Results}

The questionnaire was sent to 375 patients with patella fractures who were treated in our Level-I trauma center between the year 2003 and 2016. Overall 111 questionnaires were returned corresponding to a respond rate of $29.6 \%$. Following the inclusion criteria, 54 patients ( 20 male, 34 female) with a mean age of 63.2 years were enrolled. The remaining 57 patients were excluded because of either incomplete questionnaires $(n=13)$, conservative treatment $(n=21)$, no and shorter follow-up with X-ray control $(n=18)$, respectively, or "MQK" follow up under 6 months $(n=5)$.

The enrolled patients presented with $10 \%$ AO.34 type B and $90 \%$ AO. 34 type $\mathrm{C}$ fractures according to the AO classification. $50 \%$ of the cases were treated with tension band wiring, in $20 \%$ screw fixations, in $13.3 \%$ tension band wiring with screw fixation and in $6.6 \%$ locking plate fixation (Starplate ${ }^{\circledR}$, Arthrex) was performed. In $58.1 \%$ of the patients implant removal was performed due to either symptomatic irritation by the implant or prominence of the implant. Eight patients suffered from postoperative complaints in terms of short follow-up implant failure so that revision surgery needed to be performed. Five of these cases were treated with tension band wiring, one patient suffered from a dislocation of the inferior patella pole after locking plate fixation and one patient received revisional surgery due to a plica syndrome along with implant failure of screw fixation.

Regarding the evaluation of ISR in group B the time point for the latest follow-up was set at a minimum of 6-week post-surgery. The follow-up time between group A and B accounted for a mean of $503.8 \pm 655.7$ days. The analysis of the ISR on lateral radiographs of the knee 2 days after surgery (Group A) showed $9.1 \%$ patella baja and 27.3\% patella alta. The ISR assessed on the last follow-up (group B) revealed in $20.0 \%$ cases a patella baja and in $14.5 \%$ a patella alta. Proportionally the ratio of patella baja changed from group A to group B from 1:11 to 1:5, respectively, of patella alta from 1:3.6-1:6.9 $(p=0.05)$ (see Table 1). The mean ISR of patella baja, norma and alta of both groups showed no significant difference $(p=0.3)$ (see Table 2).

The evaluation of the MKQ was considered at a time frame from more than 6 months. The mean functional outcome

Table 1 Demonstration of patella baja, norma and alta in group A and $\mathrm{B}$ and overall functional outcome of MKQ in patella baja, alta and norma

\begin{tabular}{llll}
$\begin{array}{l}\text { Patella baja } \\
(n=16)\end{array}$ & $\begin{array}{l}\text { Patella alta } \\
(n=23)\end{array}$ & Patella norma $P$ \\
\hline $5^{*}$ & $15^{*}$ & 30 & n.s. \\
$11^{*}$ & $8^{*}$ & 36 & n.s.* \\
$65.4 \% \pm 20.8$ & $69.0 \% \pm 18.2$ & $61.8 \% \pm 13.5$ & 0.2 \\
\hline
\end{tabular}

Table 2 Mean ISR of group A and group B in patella baja, alta and norma

\begin{tabular}{lllll}
\hline Mean ISR & Patella baja & Patella alta & Patella norma & $P$ \\
\hline Group A & $0.74 *$ & $1.36^{*}$ & 1.01 & n.s.* \\
Group B & $0.73^{*}$ & $1.39^{*}$ & 0.99 & n.s.* \\
\hline
\end{tabular}

revealed relatively good general results with a mean of $65.1 \% \pm 14.9$ in the MKQ. In detail, patients with an AO.34 type B presented a mean MKQ score of $74.5 \% \pm 11.0$ and AO.34 type $\mathrm{C}$ fractures with a mean of $64.0 \% \pm 15.0$. There was no significant different functional outcome assessed by the MKQ in patients with type $\mathrm{B}$ or $\mathrm{C}$ fractures $(p=0.1)$.

The functional outcome according to the MKQ accounted for patients with patella baja for $67.1 \% \pm 17.9$ and for patella alta for $69.0 \% \pm 18.2$. The overall MKQ Score with normal ISR was $62.2 \% \pm 14.1$.

No significant difference in functional outcome in the MKQ resulted comparing patella baja, norma and alta ( $p=0.9$, see Fig. 2).

\section{Discussion}

The purpose of this retrospective study was to evaluate the change in patella height and functional outcome in patella fractures. Generally speaking several surgical treatment options are available according to the fracture type using the AO classification. Open or closed fixation using tension band wiring with its modifications as well established techniques for transverse fractures of the patella are widely used [17-19]. Dy et al. reported in their meta-analysis about several complications of tension band wiring resulting in a less functional outcome and high revision rates in up to one-third of all patients including loss of reduction and secondary

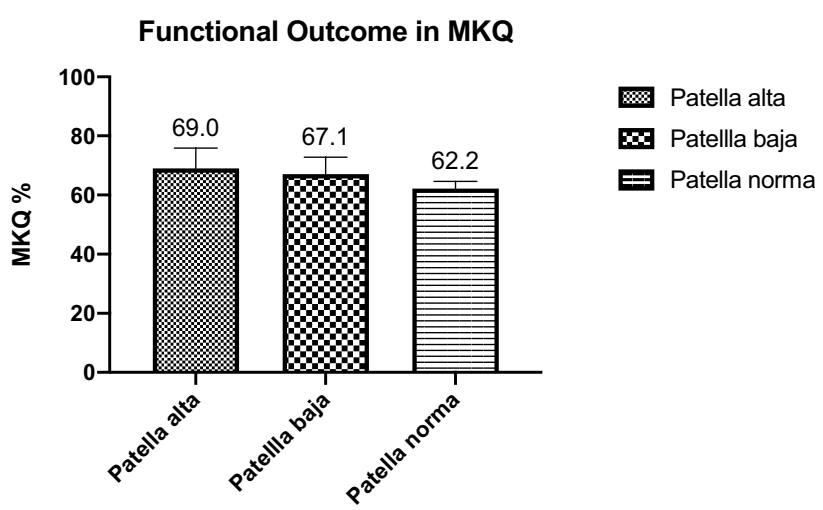

Fig. 2 Functional outcome in the mean MKQ score assessed on the latest follow-up is shown for all patella heights: patella baja, alta and norma $(p=0.2)$ 
dislocation, implant failure or implant discomfort [20, 21]. There are several studies in the actual literature on the functional outcome of the knee joint following patella fracture. In this context Wurm et al. focused on the locking plate fixation treatment [22-25] commonly postulating good results for locking plate fixation in the treatment of comminuted patella fractures. Furthermore, Ellwein et al. reported about restored function of the knee within 6 months after surgery [24]. To the best of our knowledge the presented study shows for the first time results on the functional outcome in refer to the patella height following surgically treated patella fractures. Our results demonstrate that different postoperative patella heights apparently do not influence the functional outcome.

In general especially a patella baja position is conjoined with more stress related complaints, since the lower height of the patella can lead to structural changes of the patellar tendon and functional weakness of the quadrizeps muscle correspondingly [26]. In addition, congenital soft tissue disorders, posttraumatic or postoperative changes of the soft tissue due to scaring and tissue adherence are supposed to play a key roll in anterior knee pain syndromes [27, 28]. In the literature an infra-patellar contracture syndrome or premature osteoarthritis due to patellofemoral misalignment was already well described decades ago but still undergoes intensive research [29, 30]. Also the surgical approach might simultaneously result in a patella baja and its corresponding complications in terms of either impingement or a lower lever arm along with decreased range of motion [31]. In this context our results show a proportional decrease of patella height, since more cases of patella baja and less patella alta were found in total at the latest follow-up. In our opinion this might be triggered by evolution of adhesive or scar tissue after surgery. Another reason may be the postoperative rehabilitation program including a limited range of motion of the knee joint for several weeks. However, so far, the radiological finding of a patella baja was not clinically proven in changes of the functional outcome, but the presented results of our study with a relatively short follow-up time show that patella height does not influence postoperative functional outcome.

For the retrospective assessment of the functional outcome following patella fracture the well-known Munich Knee Questionnaire (MKQ) was used. The MKQ presents a self-assessment test allowing for a subjective and objective patient-based evaluation of post-therapeutic problems of the knee. In the presented study, patients suffering from either a postoperative patella alta or baja showed good to relatively good functional outcome in the MKQ. The functional outcome showed no significant difference between patients with patella baja, norma and alta. Moreover, no noticeable difference in the functional outcome was found in relation to the severity of the patella fracture according to the AO classification. In the current literature corresponding studies reveal good results in the SF-36/KOOS Score and Special
Surgery Knee Scoring (SSKS) after surgical treatment of patella fractures [32] [33, 34], so it can be concluded that the recent literature reflects the presented results of the MKQ after surgical therapy of patella fractures.

The actual study presents with several limitations. First of all due to the retrospective study design a selection bias was accepted. The high dropout rate of all screened patients, who were treated for patella fractures between 2003 and 2016 may alter the results of functional outcome. This circumstance may influence the functional outcome proportionally, as patients with bad functional outcome may have not been considered in the general results. A subgroup analysis of functional outcome in different surgical technique was not performed, as the number of patients per performed procedure was too low in total to achieve statistically reliable results. This may have influenced the functional outcome of different fracture types. The high drop-out rate of our initially screened 375 patients is mainly explained by the restricted answer rate $(<30 \%)$ of the MKQ along with the inclusion criteria of a minimum follow-up for the ISR change and MKQ. Furthermore, only postoperative changes were evaluated. We consider the high drop-out rate of the initially screened patients as a problem of inconsistent patients' answer in this long observation interval and restricted utilization of incorrect MKQs. This sampling bias may influence the results by not including bad functional outcomes of these patients. We accept this selection bias as a concern in our results.

Due to the retrospective design of the presented study but also due to radiation exposure reasons the patella height of the contralateral, healthy knee joint was not considered. This may have restricted the incidence of a preexisting patella baja or alta and may have influenced the results of postoperative change in patella height.

In addition the authors have to admit that the evaluation of the patella height differs significantly between the different scoring systems. The interobserver reliability on plain lateral radiographs is low; therefore, we used the ISR as a well established method without high interobserver differences, to discriminate between patella baja, norma and alta [35]. But this in fact, may also influence the incidence of patella baja or alta in our patient cohort.

\section{Conclusion}

Restoring the extensor mechanism of the knee joint still remains crucial in treatment of patella fractures. In this context the common literature believes that different levels of patella height have an influence on anterior knee pain. According to our results, the patella height does not significantly affect the functional outcome following surgical fracture treatment. In addition, our data demonstrate well that there is also no 
significant difference of the functional outcome referring to the severity of the patella fracture using the AO classification.

Funding Open Access funding enabled and organized by Projekt DEAL.. No external funding was requested.

\section{Declarations}

Conflict of interests The authors declare that there are no financial or personal conflicts of interests that could have influenced this work.

Ethics approval The study was approved by the Institutional Review Board (IRB approval; reference number 409/15 s).

Open Access This article is licensed under a Creative Commons Attribution 4.0 International License, which permits use, sharing, adaptation, distribution and reproduction in any medium or format, as long as you give appropriate credit to the original author(s) and the source, provide a link to the Creative Commons licence, and indicate if changes were made. The images or other third party material in this article are included in the article's Creative Commons licence, unless indicated otherwise in a credit line to the material. If material is not included in the article's Creative Commons licence and your intended use is not permitted by statutory regulation or exceeds the permitted use, you will need to obtain permission directly from the copyright holder. To view a copy of this licence, visit http://creativecommons.org/licenses/by/4.0/.

\section{References}

1. Galla M, Lobenhoffer P (2005) Der Chirurg; Zeitschrift fur alle Gebiete der operativen Medizen. Patella fractures 76(10):987-997

2. Galla M, Lobenhoffer P (2005) Frakturen der Patella. Der Chirurg 76(10):987-999

3. Carpenter JE et al (1997) Biomechanical evaluation of current patella fracture fixation techniques. J Orthop Trauma 11(5):351-356

4. Weber $\mathrm{M}$ et al (1980) Efficacy of various forms of fixation of transverse fractures of the patella. J Bone Joint surg am 62(2):215-220

5. Wurm S, Bühren V (2015) Patellafrakturen. Trauma und Berufskrankheit 17(3):153-159

6. Wild $\mathrm{M}$ et al (2010) Fixed-angle plate osteosynthesis of the patella-An alternative to tension wiring? ClinBiomech 25(4):341-347

7. Thelen $\mathrm{S}$ et al (2012) Biomechanical cadaver testing of a fixedangle plate in comparison to tension wiring and screw fixation in transverse patella fractures. Injury 43(8):1290-1295

8. Miller MA et al (2012) Factors predicting failure of patella fixation. J Trauma Acute Care Surg 72(4):1051-1055

9. Levack B, Flannagan J, Hobbs S (1985) Results of surgical treatment of patellar fractures. Bone Joint J 67(3):416-419

10. Wild M, Windolf J, Flohé S (2010) Patellafrakturen. Unfallchirurg 113(5):401-412

11. Dye SF (2005) The pathophysiology of patellofemoral pain: a tissue homeostasis perspective. ClinOrthopRelat Res 436:100-110

12. Luyckx T et al (2009) Is there a biomechanical explanation for anterior knee pain in patients with patella alta? Bone Joint $\mathbf{J}$ 91(3):344-350

13. Gold GE et al (2004) Weight-bearing MRI of patellofemoral joint cartilage contact area. J MagnResonImag 20(3):526-530
14. Insall J, Salvati E (1971) Patella Position in the Normal Knee Joint 1. Radiology 101(1):101-104

15. Caton J et al (1982) Apropos of 128 cases. Revue de chirurgieorthopediqueetreparatrice de l'appareilmoteur. Patella infera 68(5):317-325

16. Beirer M et al (2015) The Munich Knee Questionnaire: development and validation of a new patient-reported outcome measurement tool for knee disorders. Arthroscopy J Arthroscopic Related Surg 31(8):1522-1529

17. Zhang $\mathrm{Y}$ et al (2018) Efficacy of K-wire tension band fixation compared with other alternatives for patella fractures: a metaanalysis. J OrthopSurg Res 13(1):226

18. Müller E, Frosch K (2019) Der ChirurgZeitschrift fur AlleGebiete der OperativenMedizen. Fractures of the patella 90(3):243-254

19. Kim K-S et al (2020) Suture anchor fixation of comminuted inferior pole patella fracture-novel technique: suture bridge anchor fixation technique. Arch Orthopaedic Trauma Surg 1-9

20. Dy CJ et al (2012) Meta-analysis of re-operation, nonunion, and infection after open reduction and internal fixation of patella fractures. J Trauma Acute Care Surg 73(4):928-932

21. Sim J-A et al (2020) Patellar fractures in elderly patients: a multicenter computed tomography-based analysis. Arch Orthop Trauma Surg 1-7

22. Wurm S, Bühren V, Augat P (2018) Treating patella fractures with a locking patella plate-first clinical results. Injury 49:S51-S55

23. Moore TB et al (2018) Fixed angle plate fixation of comminuted patellar fractures. Injury 49(6):1203-1207

24. Ellwein A et al (2019) Outcomes after locked plating of displaced patella fractures: a prospective case series. IntOrthop 43(12):2807-2815

25. Vedel JO et al (2018) Altered long-term health-related quality of life in patients following patella fractures: a long-term follow-up study of 49 patients. Eur J Trauma EmergSurg 44(5):707-716

26. Tecklenburg K et al (2006) Bony and cartilaginous anatomy of the patellofemoral joint. Knee Surg Sports TraumatolArthrosc 14(3):235-240

27. Noyes FR, Wojtys EM, Marshall MT (1991) The early diagnosis and treatment of developmental patella infera syndrome. ClinOrthopRelat Res 265:241-252

28. Cutbill JW et al (1997) Anterior knee pain: a review. Clin J Sport Med 7(1):40-45

29. Paulos LE et al (1987) Infrapatellar contracture syndrome: an unrecognized cause of knee stiffness with patella entrapment and patella infera. Am J Sports Med 15(4):331-341

30. Macri EM et al (2020) Relation of patellofemoral joint alignment, morphology, and radiographic osteoarthritis to frequent anterior knee pain: data from the multicenter osteoarthritis study. Arthritis Care Res 72(8):1066-1073

31. Chonko D, Lombardi A Jr, Berend K (2004) Patella baja and total knee arthroplasty (TKA): etiology, diagnosis, and management. SurgTechnolInt 12:231-238

32. LeBrun CT, Langford JR, Sagi HC (2012) Functional outcomes after operatively treated patella fractures. J Orthop Trauma 26(7):422-426

33. Berg EE (1997) Open reduction internal fixation of displaced transverse patella fractures with figure-eight wiring through parallel cannulated compression screws. J Orthop Trauma 11(8):573-576

34. Modified Hospital for Special Surgery Knee Scoring System (2006) J Orthop Trauma 20(8):S100-S101

35. Seil $\mathrm{R}$ et al (2000) Reliability and interobserver variability in radiological patellar height ratios. Knee Surg Sports Traumato1Arthrosc 8(4):231-236

Publisher's Note Springer Nature remains neutral with regard to jurisdictional claims in published maps and institutional affiliations. 